\title{
Convergence to Equilibrium in a System of Reacting Polymers *
}

\author{
Michael Aizenman ${ }^{1}$ and Thor A. Bak ${ }^{2}$ \\ 1 Department of Physics, Princeton University, Princeton, New Jersey 08540, USA \\ ${ }^{2}$ Chemistry Laboratory III, University of Copenhagen, DK-2100 Copenhagen, Denmark
}

\begin{abstract}
Chemical kinetics of a system of reacting polymers is modelled by an equation which shares certain properties with Boltzmann's equation. Being more tractable, however, this evolution may be of an illustrative value for the latter. The existence and uniqueness of solutions are analysed. We derive an entropy production inequality which is used to prove global exponential decay of the free energy. With its aid a uniform rate for strong convergence to equilibrium is proven. The generators of the linearized flow at the vicinity of the equilibria are diagonalized.
\end{abstract}

\section{Introduction}

Many substances form long-chain polymers of varying length. The distribution of the length of the polymers is determined by the dynamical equilibrium between competing reactions; that of degradation, caused by the breaking of bonds, and recombination in which two linear polymers join at their ends.

In a simple model of such a system the density function (whose argument is the length of a polymer) obeys a dynamical equation which shares certain properties with Boltzmann's equation. However, as it turns out, this equation is more amenable to analysis and as such it may be of illustrative value. In particular, it offers an example in which an analog of the $H$-theorem can be used directly, with the aid of a new inequality, to prove a global convergence to equilibrium.

The following notation is being used:

$\hat{c}(t, n)$ is the number of polymers of $n$ units; $A$ is a quantity of the order of magnitude of the total number of polymers (e.g. Avogadro number): $\delta$ is the length of a building unit of the polymers; $x=n \cdot \delta$ is the length of a polymer; $c(x)=\hat{c}(x / \delta) \delta^{-1} A^{-1}$, i.e. $c(x) d x$ is the number, in the units of $A$, of polymers whose length is in $[x, x+d x)$.

Assuming that all the bonds (of which there are $n-1$ in a polymer of length $n$ ) break independently and with the same rate and that the probability for two

* Work partly supported by U.S. National Science Foundation grant MCS 75-21684 A02 
molecules to combine is independent of their lengths we have:

$$
\begin{aligned}
\frac{\partial}{\partial t} \hat{c}(t, n)= & \hat{K}_{1} 2 \sum_{n+1}^{x} \hat{c}(t, m)-\hat{K}_{1}(n-1) \hat{c}(t, n) \\
& +\hat{K}_{2} \sum_{0}^{n} \hat{c}(t, n-m) \hat{c}(t, m)-\hat{K}_{2} 2 \hat{c}(t, n) \sum_{0}^{\infty} \hat{c}(t, m) .
\end{aligned}
$$

$\hat{K}_{1}$ and $\hat{K}_{2}$ are the reaction rates for degradation and recombination, respectively. In order for the reaction time of a long molecule to be finite, both $\hat{K}_{1}$ and $\hat{K}_{2}$ should be small. We define the rescaled reaction constants $K_{1}$ and $K_{2}$ by

$$
\hat{K}_{1}=K_{1} \delta, \quad \hat{K}_{2}=K_{2} / A \text {. }
$$

In the Riemann approximation, $\delta \sum_{n} \approx \int d x$, Eq. (1.1) leads to:

$$
\begin{aligned}
\frac{\partial}{\partial t} c(t, x)= & K_{1} 2 \int_{x}^{\infty} d y c(t, y)-K_{1} x c(t, x) \\
& +K_{2} \int_{0}^{x} d y c(t, x-y) c(t, y)-K_{2} 2 c(t, x) \int_{0}^{\infty} d y c(t, y) .
\end{aligned}
$$

Since the quantity $\bar{c}(x)=a c(b x)$ obeys a similar equation with $K_{1}$ and $K_{2}$ replaced by

$$
\bar{K}_{1}=K_{1} b, \quad \bar{K}_{2}=K_{2} b / a
$$

it is enough to study the case $K_{1}=K_{2}=1$ :

$$
\begin{aligned}
\frac{\partial}{\partial t} c(t, x)= & 2 \int_{x}^{\infty} d y c(t, y)-x c(t, x)+(c * c)(t, x) \\
& -2 c(t, x) \int_{0}^{\infty} d y c(t, y) .
\end{aligned}
$$

In the above convolution $c$ is to be treated as a function which vanishes on $(-\infty, 0)$.

Equation (1.1) was formulated by Blatz and Tobolsky [1], whose rate constant $K_{2}$ differs from ours by a geometric factor of 2 . Their discussion was restricted to a particular solution which corresponds to a solution of Eq. (1.5) of the form

$$
c(t, x)=\alpha(t)^{2} \exp [-\alpha(t) x] .
$$

This is a solution provided

$$
\frac{d}{d t} \alpha(t)=1-\alpha(t)^{2}
$$

that is $[$ for $\alpha(0)>0]$

$$
\alpha(t)=\left\{\begin{array}{cr}
\tanh \left(t+t_{0}\right) & 0<\alpha(0)<1 \\
1 & \alpha(0)=1 \\
\operatorname{coth}\left(t+t_{0}\right) & \alpha(0)>1
\end{array}\right.
$$

with some $t_{0}>0$. 
The above is a particular solution, albeit a very interesting one since it shows that the simple form of (1.6) is preserved by the time evolution and it exhibits convergence to a stationary distribution:

$$
c(t, x) \underset{(t \rightarrow \infty)}{\longrightarrow} e^{-x} .
$$

In fact, all the exponential distributions, $e^{-\lambda x}$ with $\lambda>0$, are stationary for Eq. (1.5) and form a class of what Flory [2] named the most probable distributions. Neither his paper nor the later work of Blatz and Tobolsky clarify this concept.

Apart from the fundamental questions of the existence, uniqueness and positivity of solutions for general initial data, (1.9) suggests the question of global convergence to equilibrium. Formally, the quantity $\int_{0}^{\infty} d x x_{c}(t, x)$ is a constant of the motion, as the total number of units, to which it corresponds, should be. One could therefore ask whether any solution converges to the corresponding exponential distribution selected by this conservation law.

Equation (1.1) has been studied before by one of us [3] in connection with the experimental determination of the rate of degradation of very large molecules. Reference to earlier work on that problem can be found in that paper. The linearized equation, and in particular its discrete version, which is also being studied by Kjær [4], is of interest in connection with the experimental study of the approach to equilibrium in systems containing hydrogen-bounded oligomers [5].

In the present paper we discuss the dynamics generated by Eqs. (1.1) and (1.5). The existence and uniqueness of solutions are discussed in Sect. II, where some spurious solutions are also exhibited. In Sect. III it is shown rigorously that, in analogy with Boltzmann's $H$-theorem, the free energy of the system is nonincreasing. This result is strengthened in Sect. IV where, with the aid of a new inequality, the free energy is proven to decrease, exponentially fast, all the way to its equilibrium value. This provides a necessary step in Boltzmann's method of proof of convergence to equilibrium, which in general is not easily accomplished. In Sect. $\mathrm{V}$ the decay of the free energy is being used to prove uniform convergence to equilibrium for a general class of initial data. In Sect. VI the spectra of the linearized generator, at the equilibrium points, are given exactly. Finally, the exact solution for the time-dependent Laplace transform of $c$ is given in the Appendix. The explicit solution is not easily invertible and does not seem to offer a direct way of proving the above results.

In order to shorten expressions which involve functions of several variables, like $c(t, x)$, we shall occasionally omit the explicit reference to some of the variables which are fixed in a given expression.

\section{A Reformulation Which Ensures Unique Solutions}

Equation (1.5) offers an incomplete specification of the dynamics. For a given initial data it has various solutions but only one of them describes systems which are essentially finite and this is the one we shall choose. As it turns out, the singularity lies entirely in the linear part of the generator which, therefore, shall be discussed first. 


\section{a) The Linear Part}

A natural norm, which corresponds to the total number of particles, and the space of functions in which Eq. (1.5) will be discussed are

$$
\|c\|_{x}=\int_{0}^{\infty} d x x|c(x)|
$$

and

$$
\mathscr{B}=\left\{c:[0, \infty) \rightarrow \mathbb{R} \mid\|c\|_{x}<\infty\right\} .
$$

The linear part of Eq. (1.2) is given by an operator $A$ which acts as

$$
(A c)(x)=2 \int_{x}^{\infty} d y c(y)-x c(x)
$$

$\forall L>0, A$ leaves

$$
\mathscr{B}_{L}=\{f \in \mathscr{B} \mid f \equiv 0 \quad \text { on } \quad[L, \infty\}
$$

invariant and its restriction to $\mathscr{B}_{L}$ is bounded:

$$
\|A f\|_{x} \leqq 2 L\|f\|_{x} \quad \forall f \in \mathscr{B}_{L} .
$$

We shall use this fact to define the semigroup on $\bigcup_{L>0} \mathscr{B}_{L}$. A will then be defined as the generator of the extension of the semigroup to $\mathscr{B}$. In this way the invariance of $\mathscr{B}_{L}$ is manifestly ensured, reflecting the fact that molecules longer than $L$ cannot be produced by disintegration, if initially such molecules were absent.

Lemma 2.1. i) For any $L>0$ and $f \in \mathscr{B}_{L}$ the equations

$$
\left\{\begin{array}{c}
\frac{d}{d t} c(t)=A c(t) \\
c(o)=f
\end{array}\right.
$$

have a unique solution in $\mathscr{B}_{L}$.

ii) The corresponding semigroups $\alpha_{t}^{(L)}=\exp \left(t A \uparrow \mathscr{B}_{L}\right)$ are consistent: if $L_{1}>L_{2}$ then $\forall f \in \mathscr{B}_{L_{2}}$

$$
\alpha_{t}^{\left(L_{2}\right)} f=\alpha_{t}^{\left(L_{1}\right)} f
$$

iii) $\alpha_{t}$ are positivity preserving contractions.

iv) For any $L>0$ and $f \in \mathscr{B}_{L}$

$$
\int_{0}^{\infty} d x x\left(\alpha_{t}^{(L)} f\right)(x)=\int_{0}^{\infty} d x x f(x)
$$

Proof. i) and ii) follow from the existence and uniqueness of semigroups for bounded generators. $\alpha_{t}^{(L)}$ is positivity preserving since $A \uparrow \mathscr{B}_{L}$ is a sum of two bounded operators both of which generate positivity preserving semigroups. The 
last property implies also that for $f \geqq 0, f \in \mathscr{B}_{L}$,

$$
\begin{aligned}
\frac{d}{d t}\left\|\alpha_{t} f\right\|_{x} & =\frac{d}{d t} \int_{0}^{\infty} d x x f(t, x) \\
& =\int_{0}^{\infty} d x x(A f)(t, x)=0
\end{aligned}
$$

[for the second equality the $\|\cdot\|_{r}$-differentiability of $f(t)$ was used]. Thus for positive $f \in \mathscr{B}_{L}\left\|\alpha_{t}^{(L)}\right\|_{x}=\|f\|_{x}$ and in general, using the decomposition into positive and negative parts, $\left\|\alpha_{t}^{(L)} f\right\|_{x} \leqq\|f\|_{x} \forall f \in \mathscr{B}_{L}$.

We may now extend the definition of the semigroup to $\mathscr{B}$. Let $P_{L}$ be the projections on $\mathscr{B}_{L}$ defined by

$$
\left(P_{L} f\right)(x)= \begin{cases}f(x) & 0 \leqq x<L \\ 0 & L \leqq X\end{cases}
$$

\section{Proposition 2.1.}

i) $\alpha_{t}=s-\lim _{L \rightarrow \infty} \alpha^{(L)} P_{L}$

exist and form a positivity preserving contraction semigroup on $\mathscr{B}$.

ii) $\alpha_{t}$ satisfies

$\int_{0}^{\infty} d x x \alpha_{t} f(x)=\int_{0}^{\infty} d x x f(x)$

iii) The generator of $\alpha_{t}$ is the closure of $A$ restricted to

$\mathscr{D}_{0}(A)=\left\{f \in \mathscr{B}\left|\int_{0}^{\infty} d x x^{2}\right| f(x) \mid<\infty\right\}$.

iv) $\alpha_{t}$ is the unique strongly continuous semigroup whose generator satisfies Eq. (2.1) and such that $\forall L>0, \alpha_{t} \mathscr{B}_{L} \subset \mathscr{B}_{L}$.

Proof. i) Let $f \in \mathscr{B}$. Since for $\bar{L}>L$

$$
\left\|\alpha_{t}^{(\bar{L})} P_{\bar{L}} f-\alpha_{t}^{(\bar{L})} P_{L} f\right\|_{x} \leqq\left\|\left(P_{\bar{L}}-P_{L}\right) f\right\|_{x},
$$

$\mathrm{a}_{t}^{(L)} P_{L} f$ are Cauchy uniformly in $t$. Thus the limit exists uniformly in $t$ and $\alpha_{t}$ inherits the above mentioned properties of $\alpha_{t}^{(L)}$.

ii) The equality follows by continuity.

iii) On $\mathscr{B}_{L} \alpha_{t}=\alpha_{t}^{(L)}$. Thus $\alpha_{t}$ leaves $\bigcup_{L} \mathscr{B}_{L}$ invariant. The generator of $\alpha_{t}$ is therefore the closure of $A \uparrow \bigcup_{L} \mathscr{B}_{L} \quad[10]$. Since $\mathscr{D}_{0}(A) \supset \bigcup_{L} \mathscr{B}_{L} \quad$ and $\forall f \in \mathscr{D}_{0}(A) A P_{L} f \rightarrow A f, \mathscr{D}_{0}(A)$ too is a core for the generator.

iv) The uniqueness follows from the above argument which applies to any semigroup which satisfies the assumption in iv).

As to the uniqueness of individual solutions; the following criterion follows from i) and iii). 
Corollary 2.1. Let $f \in \mathscr{D}_{0}(A)$. There is at most one continuously $\|\cdot\|_{x}$-differentiable solution to

$$
\left\{\begin{array}{l}
\frac{d}{d t} c(t)=A c(t) \\
c(0)=f
\end{array}\right.
$$

such that $c(t) \in \mathscr{D}_{0}(A) \forall t \geqq 0$.

Our preoccupation with the uniqueness is not unfounded since Eq. (2.3) has additional solutions in $\mathscr{B}$, e.g.

$$
c_{a}(t, x)=e^{t a} /(x+a)^{3}
$$

with $a>0$. Thus, by adding linear combinations (in $a$ ) of

$$
\begin{aligned}
& \alpha_{t} c_{a}(0, x)-c_{a}(t, x) \\
& \quad=\left[e^{-t x}+t(x+a) e^{-t x}+\frac{1}{2} t^{2}(x+a)^{2} e^{-t x}-e^{t a}\right] /(x+a)^{3}
\end{aligned}
$$

[see Eq. (2.8)], it is seen that solutions of Eq. (2.3) are non-unique for any initial data. This however is consistent with Corollary 2.1, in particular $c_{a}(t, \cdot) \notin \mathscr{D}_{0}(A)$.

The same analysis, after the obvious modifications, applies in the discrete case (1.1), where the linear evolution is described by

$$
\frac{d}{d t} \hat{c}(t, n)=2 \hat{K}_{1} \sum_{n+1}^{\infty} \hat{c}(t, m)-(n-1) \hat{K}_{1} \hat{c}(t, n) .
$$

The semigroup is explicitly given by the following expression which was found in $[3]$,

$$
\hat{c}(t, n)=e^{-(n-1) \hat{K}_{1} t} \sum_{m}^{\infty}\left\{\left(1-e^{-2 \hat{K}_{1} t}\right)+\left(1-2 e^{-\hat{K}_{1} t}+e^{-2 \hat{K}_{1} t}\right)(m-n)\right\} \hat{c}(0, m) .
$$

In the continous case, with $K_{1}=1$, this reduces to

$$
\left(\alpha_{t} c\right)(x)=e^{-t x} c(x)+2 t e^{-t x} \int_{x}^{\infty} d y c(y)+t^{2} e^{-t x} \int_{x}^{\infty} d y(y-x) c(y) .
$$

\section{b) The Full Equation}

Equation (1.5) inherits the non-uniqueness which is present in its linear part. Having seen how to resolve this we reformulate the equation of the dynamics, using the chosen solution for the linear term, as

$$
c(t, \cdot)=\alpha_{t} c(0, \cdot)+\int_{0}^{t} d s \alpha_{t-s} K(c(s, \cdot))
$$

with

$$
K(c)(x)=(c * c)(x)-2 c(x) \int_{0}^{\infty} c(y) d y .
$$

Equation (1.2) is recovered from (2.9) by differentiation. 
We shall discuss the solutions of Eq. (2.9) in the space $\mathscr{F}$ defined as follows.

$$
\begin{aligned}
& \|c\|_{1}=\int_{0}^{\infty} d x|c(x)|, \quad\|c\|_{x}=\int_{0}^{\infty} d x x|c(x)| \\
& \|c \mid\|=\|c\|_{1}+\|c\|_{x} \\
& \mathscr{F}=\left\{f:[0, \infty) \rightarrow \mathbb{R}|\|f \mid\|<\infty\}, \quad \mathscr{F}_{+}=\{f \in \mathscr{F} \mid f(\cdot) \geqq 0\} .\right.
\end{aligned}
$$

Lemma 2.2. Let $f \in \mathscr{F}$ then $\exists T \in(0, \infty]$, such that for $t \in[0, T) E q$. (2.9) has a unique solution with $c(0, x)=f(x)$ and $c(t, x) \in \mathscr{F} \forall t \in[0, T)$. The maximal $T$ with that property, $\hat{T}$, is finite only if $\limsup _{t \rightarrow \hat{T}}\|\| c(t, \cdot) \|=\infty$.

Proof. Let $c_{1}, c_{2} \in \mathscr{F}$, then

$$
\begin{aligned}
& \|\| K\left(c_{1}\right)-K\left(c_{2}\right)\|\| \\
& \quad=\|\left(c_{1}-c_{2}\right) *\left(c_{1}+c_{2}\right)-2\left(c_{1}-c_{2} \int_{0}^{\infty} d y c_{1}(y)-2 c_{2} \int_{0}^{\infty} d y\left[c_{1}(y)-c_{2}(y)\right] \|\right. \\
& \quad \leqq 5\left(\left\|c_{1}\right\|+\|+\| c_{2} \|\right)\left(\left\|c_{1}-c_{2} \mid\right\|\right) .
\end{aligned}
$$

Therefore the map $c \mapsto K(c)$ is locally Lipschitz in $\mathscr{F}$.

Further, $\forall f \in \mathscr{F}$

$$
\left\|\alpha_{t} f\right\|_{x} \leqq\|f\|_{x}, \quad\left\|\alpha_{t} f\right\|_{1} \leqq\|f\|_{1}+t\|f\|_{x}
$$

and

$$
\left\|\alpha_{t} f\right\| \leqq(1+t)\|f\| \|
$$

[due to the linearity it is enough to verify it for non-negative $f$ for which it follows by integrating Eq. (2.8)].

Let $f \in \mathscr{F}$. It follows from (2.10) and (2.11) that for $b>\|\| f \|$, and $T>0$ such that $\left(T+T^{2} / 2\right) 10 b^{2}=(b-\|f\|)+2 / b$ the mapping

$$
\begin{aligned}
& c(t) \mapsto \bar{c}(t) \\
& \bar{c}(t)=\alpha_{t} f+\int_{0}^{t} d s \alpha_{t-s} K(c(s))
\end{aligned}
$$

is a contraction in the space of continuous functions

$$
\{c:[0, T) \rightarrow\{g \in \mathscr{F}||\|g\| \mid \leqq b\}\},
$$

equipped with the $\sup _{t}|\|c(t) \mid\|$ norm. A standard argument implies now the local existence and uniqueness of solutions of Eq. (2.9) [given by the fixed point of the mapping (2.12) with $f=c(0)]$.

Some of the solutions of Eq. (2.9) blow up in a finite time [e.g. if $N(0)<0$, see (2.13)], this however does not happen if initially $c$ is non-negative, which is the case of interest: 
Proposition 2.2. Let $f \in \mathscr{F}_{+}$. Then

i) Equation (2.9) has a unique continuous solution, $c(t, x)$, such that $c(0, x)=f(x)$ and $c(t, x) \in \mathscr{F} \forall t \geqq 0$.

ii) $\forall t \geqq 0 \quad c(t, x) \geqq 0$.

Proof. The quantities

$$
N(t)=\int_{0}^{\infty} d x x c(t, x)
$$

and

$$
M(t)=\int_{0}^{\infty} d x c(t, x),
$$

obey the autonomous differential equations which are formally obtained by integrating Eq. (1.2):

$$
\begin{aligned}
& \frac{d}{d t} N(t)=0 \\
& \frac{d}{d t} M(t)=N(t)-M(t)^{2} .
\end{aligned}
$$

These equations can be derived by differentiating the autonomous integral equations for $N$ and $M$ which are obtained by integrating Eq. (2.9). It follows that if $N(0), M(0) \geqq 0$ then both stay bounded uniformly in $t \geqq 0$. Since for positive $c$, $\|c\| \|=N+M$, in order to deduce the global existence of solutions which stay uniformly bounded it suffices to prove that the positivity of $c$ is preserved under the time evolution. The necessity, for physical reasons, of such a condition is obvious. Heuristically it seems to follow from Eq. (1.5), nevertheless it is not satisfied by some of the spurious solutions.

One obtains an expansion for the solutions of Eq. (2.9) by iteratively substituting

$$
c(t, \cdot)=\alpha_{t} c(0, \cdot)+\int_{0}^{\infty} d s \alpha_{t-s}(c(s, \cdot) * c(s, \cdot))-\int_{0}^{\infty} d s \alpha_{t-s}(2 M(s) c(s, \cdot))
$$

in its last term. This is just the norm convergent (in our case) expansion, in powers of $\int d s M(s)$, of the following expression

$$
\begin{aligned}
c(t, \cdot)= & \alpha_{t}(c(0, \cdot)) \exp \left[-2 \int_{0}^{t} d s M(s)\right] \\
& +\int_{0}^{t} d s \alpha_{t-s}(c(s, \cdot) * c(s, \cdot)) \exp \left[-2 \int_{s}^{t} d u M(u)\right] .
\end{aligned}
$$

By the same contraction mapping argument as in the proof of Lemma 2.2, the unique solution of $(2.15)$ with $c(0, \cdot)=f(\cdot)$ may be obtained by iterating the mapping which takes $c(\cdot, \cdot)$ to the expression at the right hand side of $(2.15)$. In this method, starting with $c(t, \cdot) \equiv f(\cdot)$, the solution of $(2.9)$ is constructed by manifestly positivity preserving iterations. 
Proving the existence of the time evolution we obtained the autonomous Eq. (2.13) for $N(t)$ and $M(t)$. Solving them we learn an interesting property of the dynamics.

Corollary 2.2. Let $c(0, \cdot) \geqq 0, c(0, \cdot) \in \mathscr{F} . N$ and $M$ of the solution of Eq. (2.9) are given by:

$$
\begin{aligned}
& N(t)=N(0) \\
& M(t)=\left\{\begin{array}{cc}
N(0)^{1 / 2} \tanh \left[N(0)^{1 / 2}\left(t+t_{0}\right)\right] & M(0)<N(0)^{1 / 2} \\
N(0)^{1 / 2} & M(0)=N(0)^{1 / 2} \\
N(0)^{1 / 2} \operatorname{coth}\left[N(0)^{1 / 2}\left(t+t_{0}\right)\right] & M(0)>N(0)^{1 / 2},
\end{array}\right.
\end{aligned}
$$

for some $t_{0}>0$ which may be determined from $N(0)$ and $M(0)$.

Thus while $N$ (which corresponds to the density of units) is constant, $M$ (the density of polymers) tends to an equilibrium value which depends only on $N$. The convergence to equilibrium is studied in more detail in the next sections.

\section{An F-Theorem}

A class of stationary solutions of Eq. (1.2) is given by

$$
c_{a}(x)=e^{-a x} .
$$

A solution in this class is uniquely characterized by $N\left(c_{a}\right)=a^{-2}$. In view of Corollary 2.2 one may suspect that these are the equilibrium states for the system and that the time evolution leads any state to the corresponding equilibrium, as determined by the conservation law for $N(c)$.

For Boltzmann's equation convergence to equilibrium is indicated by the celebrated $H$-theorem. A natural question is whether the time evolution considered here has an analogous property.

\section{a) A Formal Argument}

In order to clarify some of the concepts we shall first discuss a more general class of equations of which (1.5) is a particular case.

An $H$-theorem is to be expected, formally at least, for all systems whose particles interact by a balanced scattering, e.g. a two particle reaction by which a pair of particles in the states $(x, y)$ produces a pair in the states $\left(x^{\prime}, y^{\prime}\right)$ at the rate

$$
c(x) c(y) K\left(x, y \mid x^{\prime}, y^{\prime}\right) d x d y d x^{\prime} d y^{\prime},
$$

which is balanced in the sense that

$$
K\left(x, y \mid x^{\prime}, y^{\prime}\right)=K\left(x^{\prime}, y^{\prime} \mid x, y\right) .
$$

Under the generated dynamics $\int d x c(x)$ is invariant and the entropy

$$
S(c)=-\int d x c(x) \ln c(x)(\equiv-H(c))
$$

is monotone non-decreasing. 
An equally tractable situation occurs if the system undergoes the reactions $x \rightarrow\left(x^{\prime}, y^{\prime}\right)$ at the rate $c(x) \alpha K\left(x \mid x^{\prime}, y^{\prime}\right) d x d x^{\prime} d y^{\prime}$ and the opposite reactions $\left(x^{\prime}, y^{\prime}\right) \rightarrow x$ at the rate $c\left(x^{\prime}\right) c\left(y^{\prime}\right) K\left(x \mid x^{\prime}, y^{\prime}\right) d x d x^{\prime} d y^{\prime}$. The contribution of such reactions to the dynamical equation is

$$
\begin{aligned}
\frac{\partial}{\partial t} c(t, x)= & -c(t, x) \alpha \int d x^{\prime} d y^{\prime} K\left(x \mid x^{\prime}, y^{\prime}\right) \\
& +2 \int d x^{\prime} d y^{\prime} c\left(t, x^{\prime}\right) \alpha K\left(x^{\prime} \mid x, y^{\prime}\right) \\
& -2 c(t, x) \int d x^{\prime} d y^{\prime} c\left(t, x^{\prime}\right) K\left(y^{\prime} \mid x, x^{\prime}\right) \\
& +\int d x^{\prime} d y^{\prime} c\left(t, x^{\prime}\right) c\left(t, y^{\prime}\right) K\left(x \mid x^{\prime}, y^{\prime}\right) .
\end{aligned}
$$

The number of particles is not conserved and for general $\alpha$ and $K, H$ is not monotone decreasing. However, another quantity is.

In order to see the relation of the above dynamics to those described by (3.2), it is convenient to add a fictitious state, $x_{0}$, and look at the reactions $x \leftrightarrow\left(x^{\prime}, y^{\prime}\right)$ as $\left(x, x_{0}\right) \leftrightarrow\left(x^{\prime} y^{\prime}\right)$. From this point of view the only difference is that the reaction rates do not depend on the concentration of reactants in the state $x_{0}$, and $c\left(x_{0}\right)$ is replaced in (3.2) by a constant $\alpha$. Such dynamics may be viewed as a weak coupling limit of the reaction described by (3.2). In this limit the reaction rate of particles in the state $x_{0}$ is scaled by $\varepsilon$ and the degeneracy of that state [i.e. the discrete measure with respect to which the density $c\left(x_{0}\right)$ is defined] is $\varepsilon^{-1}$. Equation (3.5) is obtained if, while $\varepsilon \rightarrow 0$, the total number of particles is

$$
c\left(x_{0}\right) / \varepsilon+\int_{\left(x \neq x_{0}\right)} d x c(x)=\alpha / \varepsilon .
$$

Since the number of particles is invariant,

$$
c\left(t, x_{0}\right)=\alpha-\varepsilon \int d x c(t, x)
$$

and the total entropy may be computed from $c(x), x \neq x_{0}$ :

$$
\begin{aligned}
S & =-\varepsilon^{-1} c\left(t, x_{0}\right) \ln c\left(t, x_{0}\right)-\int d x c(t, x) \ln c(t, x) \\
& =-\varepsilon^{-1} \alpha \ln \alpha+[\ln \alpha+1] \int d x c(t, x)+0(\varepsilon)-\int d x c(t, x) \ln c(t, x) .
\end{aligned}
$$

Thus the monotonicity in time should be expected for the following quantity

$$
\begin{aligned}
F_{\alpha}(c) & \equiv \int d x c(x) \ln c(x)-[\ln \alpha+1] \int d x c(x) \\
& =-S(c)-[\ln \alpha+1] \int d x c(x) .
\end{aligned}
$$

Indeed, by a formal calculation, if $c(t, x)$ obeys (3.5), then

$$
\begin{aligned}
& \frac{d}{d t} F_{\alpha}(c(t \cdot \cdot) \\
& =-\int d x d x^{\prime} d y^{\prime} K\left(x \mid x^{\prime}, y^{\prime}\right)\left[\alpha c(x)-c\left(x^{\prime}\right) c\left(y^{\prime}\right)\right] \\
& \quad \cdot\left\{\ln [\alpha c(x)]-\ln \left[c\left(x^{\prime}\right) c\left(y^{\prime}\right)\right]\right\} \leqq 0 .
\end{aligned}
$$

The last inequality follows from the monotonicity of $\ln x$. 
Equation (1.3) is a particular case of (3.5) corresponding to

$$
\begin{aligned}
K\left(x \mid x^{\prime}, y^{\prime}\right) & =K_{2} \delta\left(x^{\prime}+y^{\prime}-x\right) \\
\alpha & =K_{1} / K_{2} .
\end{aligned}
$$

The calculation is straightforward as long as one remembers that the range of $x, x^{\prime}$ and $y^{\prime}$ is $[0, \infty)$.

Hence we expect the following to be true, with

$$
F(c)=\int_{0}^{\infty} d x c(x)[\ln c(x)-1] \text {. }
$$

Proposition 3.1. Let $c(t, x)$ be a solution of Eq. (1.5) with $c(0, \cdot) \in \mathscr{F}_{+}$, then $\forall t_{2} \geqq t_{1} \geqq 0$

$$
F\left(c\left(t_{2}, \cdot\right)\right) \leqq F\left(c\left(t_{1}, \cdot\right)\right) \text {. }
$$

Proposition 3.1 is proven in Part b) of this section. The previous discussion is, of course, not necessary in order to verify that if $c$ obeys Eq. (1.5) then formally

$$
\begin{aligned}
\frac{\partial}{\partial t} F(c)= & -\int_{0}^{\infty} d x \int_{0}^{\infty} d y[c(x+y)-c(x) c(y)] \\
& \cdot\{\ln c(x+y)-\ln [c(x) c(y)]\} \leqq 0 .
\end{aligned}
$$

It may be worth pointing out that in the application $F$ is the free energy density of the system. If $e(<0)$ is the energy of a bond, then for a chemical system, modelled by the equations, the ratio of the two rate constants is

$$
\hat{K}_{1} / \hat{K}_{2}=\exp (e / k T),
$$

where $T$ is the temperature at which the system is maintained. Substituting this in (3.9), using (3.11) and (1.2), we obtain

$$
\begin{aligned}
F(c) & =-S(c)+M(c)[\ln (\delta A)-1]-e M(c) /(k T) \\
& \approx A^{-1}\left\{-\sum \hat{c}_{n}\left[\ln \hat{c}_{n}-1\right]-e \sum \hat{c}_{n} /(k T)\right\} .
\end{aligned}
$$

This is the free energy, at the temperature $T$, since the chemical energy is

$$
E(c)=e\left(-\sum_{0}^{\infty} \hat{c}_{n}\right) \approx-e A M(c),
$$

relative to the state in which all the units form a single linear polymer molecule.

\section{b) Proof of the F-Theorem}

In order to use the formal derivation of Eq. (3.14) we introduce approximating dynamics for which (3.14) is easily justified. The $F$-theorem follows by a semicontinuity argument. 
The approximating dynamics, obtained by suppressing the reactions which involve molecules longer than $z$, are described by the following equation.

$$
\frac{\partial}{\partial t} c(t, x)= \begin{cases}2 \int_{x}^{z} d y c(y)-x c(x)+(c * c)(x)-2 c(x) \int_{0}^{z-x} d y c(y) & 0<x \leqq z \\ 0 & z<x .\end{cases}
$$

Let $\alpha_{t}^{z}$ be the semigroup on $\mathscr{F}$ whose generator is the bounded operator described by the first two terms in (3.18), and let $K^{z}(c)$ be defined by the last two terms in that equation.

Since

$$
\begin{aligned}
\alpha_{t}^{z}(c) & =\left(1-P_{z}\right) c+\alpha_{t}\left(P_{z} c\right) \\
& =\alpha_{t}(c)+\left(1-P_{z}\right) c-\alpha_{t}\left(\left(1-P_{z}\right) c\right)
\end{aligned}
$$

we have [using (2.11)]:

Lemma 3.1. $\alpha_{t}^{z}$ is a positivity preserving semigroup on $\mathscr{F}$ and $\forall c \in \mathscr{F}, t \geqq 0$ :

$$
\begin{aligned}
& \left\|\alpha_{t}^{z} c \mid\right\| \leqq(1+t)\|c\| \| \\
& \left\|\alpha_{t} c-\alpha_{t}^{z} c\right\| \mid \leqq(2+t)\left\|\left(1-P_{z}\right) c\right\| \| .
\end{aligned}
$$

In analogy with our interpretation of Eq. (1.5), we shall study Eq. (3.18) using its integrated form:

$$
c_{z}(t, \cdot)=\alpha_{t}^{z} c(0, \cdot)+\int_{0}^{t} d s \alpha_{t-s}^{z} K^{z}\left(c_{z}(s, \cdot)\right) .
$$

Lemma 3.2. For a given $c_{z}(0, \cdot) \in \mathscr{F}_{+}$Eq. (3.20) has a unique solution in $\mathscr{F}_{+} \cdot$ It satisfies

$$
\left\|c_{z}(t, \cdot)\right\||\leqq(1+t)\|\mid c(0, \cdot)\| \|
$$

Proof. Let $b>\|\| c_{z}(0, \cdot)\|\|$ and let $T$ be the positive solution of $\left(T+T^{2} / 2\right) 10 b$ $=\left(b-\left\|c_{z}(0, \cdot)\right\| \|\right)+2 / b$. We define the mapping $R_{z, c(0)}$ in the Banach space of continuous functions $f:[0, T] \rightarrow \mathscr{F}$ by:

$$
\left(R_{z, c(0)} f\right)(t, x)=\alpha_{t}^{z} c_{z}(0, \cdot)+\int_{0}^{t} d s \alpha_{t-s}^{z} K^{z}(f(s, \cdot)) .
$$

By estimates analogous to those used to prove Lemma 2.2 it follows that

$$
\begin{aligned}
& \sup _{[0, T]}\left\|\left(R_{z, c_{z}(0)} f\right)(t, \cdot)-\left(R_{z, c_{z}(0)} g\right)(t, \cdot) \mid\right\| \\
& \quad \leqq \lambda \sup _{s \in[0, T]}\|\| f(s, \cdot)-g(s, \cdot)\|\|
\end{aligned}
$$

with some $\lambda<1$ which is independent of $z$. Thus for any $f$ in the above Banach space $R_{z, c(0)}^{n} f$ converge to the unique fixed point of $R_{z, c(0)}$, which is the local solution of (3.20). 
The positivity of the solution and the global existence follow by the argument given in the proof of Proposition 2.2. Equation (2.18) are, however, replaced by:

$$
\begin{aligned}
\frac{d}{d t} \int_{0}^{\infty} d x x c_{z}(t, x & =0 \\
\frac{d}{d t} \int_{0}^{\infty} d x c_{z}(t, x) & =\int_{0}^{z} d x x c_{z}(t, x)-\int_{0<x+y<z} d x d y c(x) c(y) \\
& \leqq \int_{0}^{\infty} d x x c_{z}(t, x)=\int_{0}^{\infty} d x x c_{z}(0, x) .
\end{aligned}
$$

These follow by integrating Eq. (3.18), since the generator of $\alpha_{t}^{z}$ is bounded and thus solutions of Eq. (3.20) are $\||\cdot|\|$-differentiable in $t$.

Lemma 3.3. Let $c(t, x)$ be a solution of Eq. (2.9) and let $c_{z}(t, x)$ be solutions of Eq. (3.20) with $c(0, \cdot), c_{z}(0 \cdot \cdot) \in \mathscr{F}_{+}$and $\left\|c_{z}(0, \cdot)-c(0, \cdot)\right\| \underset{(z \rightarrow \infty)}{\longrightarrow} 0$. Then for any $t \geqq 0$ $\left\|c_{z}(t, \cdot)-c(t, \cdot)\right\| \rightarrow 0$ as $z \rightarrow \infty$.

Proof. Dividing the time in intervals, it is enough to prove that for any $b>0 \exists T$ $=T(b)>0$ such that if $c$ is a solution of Eq. (2.9) and $d_{z}$ is a solution of Eq. (3.20), with $\|c(0, \cdot)\|\|,\| d_{z}(0, \cdot) \| \equiv b / 2$, then

$$
\sup _{t \in[0, T]}\left\|c(t, \cdot)-d_{z}(t, \cdot)\right\| \leqq a\left\|c(0, \cdot)-d_{z}(0, \cdot)\right\|+\varepsilon(z ; c, T)
$$

with some $a<\infty$ and a $d_{z}$-independent function $\varepsilon$ for which

$$
\lim _{z \rightarrow 0} \varepsilon(z ; c, T)=0 \text {. }
$$

Let now $T$ and $R_{z, c(0)}$ be as in the proof of Lemma $3.2\left(d(0)=d_{z}(0, \cdot)\right)$. Then

$$
d_{z}(t, \cdot)=\lim _{n \rightarrow \infty}\left(R_{z, d(0)}^{n} c\right)(t, \cdot)
$$

and, using (3.21),

$$
\begin{aligned}
& \sup _{t \in[0, T]}\left\|d_{z}(t, \cdot)-c(t, \cdot)\right\| \\
& \quad=\sup _{t \in[0, T]}\left\|\sum_{n=0}^{\infty}\left(R_{z, d(0)}^{n+1} c-R_{z, d(0)}^{n} c\right)(t, \cdot)\right\| \\
& \leqq(1-\lambda)^{-1} \sup _{t \in[0, T]}\left\|\left(R_{z, d(0)} c\right)(t, \cdot)-c(t, \cdot)\right\| .
\end{aligned}
$$

To get a bound for the right hand side we subtract (2.9) from (3.21). Thus

$$
\begin{aligned}
& \left\|\left(R_{z, d(0)} c\right)(t, \cdot)-c(t, \cdot)\right\| \mid \\
& \leqq\left\|\alpha_{t}^{z} d(0, \cdot)-\alpha_{t} c(0, \cdot)\right\|\left\|+\int_{0}^{t} d s\right\|\left\|\alpha_{t-s}^{z} K^{z}(c(s, \cdot))-\alpha_{t-s} K(c(s, \cdot))\right\| \| \\
& \leqq\left\|\alpha_{t}^{z}\left(d_{z}(0, \cdot)-c(0, \cdot)\right)\right\|\|+\|\left(\alpha_{t}-\alpha_{t}^{z}\right) c(0, \cdot)\|\| \\
& +\int_{0}^{t} d s\left|\left\|\alpha_{t-s}^{z}\left|K^{z}(c(s, \cdot))-K(c(s, \cdot))\right|\right\|\right| \\
& +\int_{0}^{t} d s\left\|\left(\alpha_{t-s}^{z}-\alpha_{t-s}\right) K(c(s, \cdot))\right\| \| \text {. }
\end{aligned}
$$


With the bounds of Lemma 3.1 we thus get

$$
\begin{aligned}
& \sup _{t \in[0, T]}\left\|d_{z}(t, \cdot)-c(t, \cdot)\right\| \| \\
& \quad \leqq(1-\lambda)^{-1}(1+T)\|d(0, \cdot)-c(0, \cdot)\|+\varepsilon(z, c, T)
\end{aligned}
$$

with

$$
\begin{aligned}
\varepsilon(z, c, T)= & (1-\lambda)^{-1} \int_{0}^{T} d s(1+t-s)\left\|K^{z}(c(s, \cdot))-K(c(s, \cdot))\right\| \| \\
& +(1-\lambda)^{-1} \int_{0}^{T} d s(2+t-s)\left\|\left(1-P_{z}\right) K(c(s, \cdot))\right\| \|
\end{aligned}
$$

$\|c(s, \cdot)\| \|$ is bounded uniformly in $s$, and by (2.10) so are $\left\|K^{z}(c(s, \cdot))\right\|$ and $\|K(c(s, \cdot))\| \|$. Therefore, (3.24) follows by the bounded convergence theorem.

To prove Proposition 3.1 we shall also need:

Lemma 3.4. Let $f, f_{n} \in \mathscr{F}_{+},\left\|f_{n}-f\right\|_{1} \underset{(n \rightarrow \infty)}{\longrightarrow} 0$. Then

$\lim \inf F\left(f_{n}\right) \geqq F(f)$.

( $F$ is lower-semi-continuous on $\mathscr{F}_{+}$.)

Proof. Applying Jensen's inequality to

$$
\int d x g(x)[f(x) / g(x)] \ln [f(x) / g(x)]
$$

one can prove that (with $h=\ln g$ )

$$
\begin{aligned}
F(f)= & {\left[\int_{0}^{\infty} d x f(x)\right]\left[\ln \int_{0}^{\infty} d y f(y)-1\right] } \\
& +\sup \left\{\int_{0}^{\infty} d x f(x) h(x) \mid h \geqq 0, \sup h<\infty, \int_{0}^{\infty} d y e^{h(y)}=1\right\} .
\end{aligned}
$$

Since $F$ in $\mathscr{F}_{+}$is a supremum over $\|\cdot\|_{1}$-continuous functions, it itself is lowersemi-continuous.

Proof of Proposition 3.1. Let $z<\infty$. If $c_{z}(t, \cdot)$ is a solution of Eq. (3.18) with

$$
c_{z}(0, \cdot) \in \mathscr{F}_{+} \text {and } \sup _{x \in[0, z]} c(0, x)<\infty,
$$

then $\forall T<\infty$ :

$$
\sup _{\substack{x \in[0, z] \\ t \in[0, T]}} c_{z}(t, x)<\infty .
$$

In such a case the formal derivation gives the correct answer, and similarly to Eq. (3.14),

$$
\begin{aligned}
\frac{d}{d t} F\left(c_{z}(t, \cdot)\right)= & -\int_{0<x+y<z} d x d y[c(x+y)-c(x) c(y)] \\
& \cdot\{\ln c(x+y)-\ln [c(x) c(y)]\} \leqq 0 .
\end{aligned}
$$


Let now $c(t, \cdot)$ be a solution of Eq. (2.9). We may assume that $F(c(0, \cdot))<\infty$, otherwise (3.10) is trivially true. One may always choose a sequence of functions $c_{z}(0, \cdot)$, with $z=1,2, \ldots$, which obey $(3.30)$ and

$$
\begin{aligned}
& \|\| c_{z}(0, \cdot)-c(0, \cdot)\|\| \underset{(z \rightarrow \infty)}{\underset{(}{\longrightarrow} 0} \\
& F\left(c_{z}(0, \cdot)\right) \underset{(z \rightarrow \infty)}{\longrightarrow} F(c(0, \cdot)) .
\end{aligned}
$$

By Lemma 3.3, $\forall t \geqq 0$ :

$$
c(t, \cdot)=\lim _{z \rightarrow \infty} c_{z}(t, \cdot) .
$$

Therefore, by Lemma 3.4 and (3.32),

$$
\begin{aligned}
F(c(t, \cdot)) & \leqq \liminf _{z \rightarrow \infty} F\left(c_{z}(t, \cdot)\right) \\
& \leqq \liminf _{z \rightarrow \infty} F\left(c_{z}(0, \cdot)\right)=F(c(0, \cdot)),
\end{aligned}
$$

which proves the proposition.

All the results of this section extend, after the obvious modifications, to the discrete case described by Eq. (1.1).

\section{Exponential Decay of the Excess Free Energy}

The free energy, $F$, is bounded below. Let us define:

$$
\hat{F}(N, M)=\inf \left\{F(c) \mid c \in \mathscr{F}_{+}, N(c)=N, M(c)=M\right\} .
$$

Proposition 4.1. $F$ is well defined on $\mathscr{F}_{+}$and

i) $\hat{F}(N, M)=M \ln \left(M^{2} / N\right)-2 M$.

ii) The unique F-minimalizing state, for specified $N$ and $M$ is given by

$C_{N, M}(x)=M^{2} / N \exp (-M / N x)$.

iii) For any $N>0$,

$$
\inf \{\hat{F}(N, M) \mid M>0\}=\hat{F}\left(N, N^{1 / 2}\right) .
$$

Proof. For $c(\cdot) \in \mathscr{F}_{+}$and $N=N(c), M=M(c)$ :

$$
\int d x c_{N, M}(x) \ln c_{N, M}(x)=\int d x c(x) \ln c_{N, M}(x) .
$$

Thus

$$
\begin{aligned}
F(c)-F\left(c_{N, M}\right) & =-\int d x c(x) \ln \left[c(x) / c_{N, M}(x)\right] \\
& =\int d x c_{N, M}(x) \Psi\left(c(x) / c_{N, M}(x)\right)
\end{aligned}
$$


with $\Psi(z)=-z \ln z$. Since $\Psi$ is bounded below $F(c)$ is well defined, possibly $+\infty$. By the convexity of $\Psi$, and Jensen's inequality,

$$
\begin{aligned}
& F(c)-F\left(c_{N, M}\right) \\
& \quad \geqq \Psi\left(\int d x c_{N, M}(x)\left[c(x) / c_{N, M}(x)\right] / \int d \bar{x} c_{N, M}(\bar{x})\right) \int d y c_{N, M}(y) \\
& \quad=\Psi(1) M=0 .
\end{aligned}
$$

The inequality is strict unless $c \equiv c_{N, M}$.

This proves ii) which directly implies i) and iii).

Since $F(c(t))$ is a decreasing function of $t$ which is bounded below it has a limit. Had it been known that this limit is the equilibrium value of $F$, under the constraint of $N$, it could have been deduced that $c(t)$ converges strongly to the equilibrium. This method is due to Boltzmann, however so far it has not been easy to apply it to Boltzmann's equation.

We shall now prove, with the aid of a new inequality, that $F(c(t))$ does decrease all the way to its equilibrium value, in fact exponentially fast, provided initially it is finite.

Let $c(t)$ be a solution of Eq. (2.9) with $c(0) \in \mathscr{F}_{+}$and let $N(t)=N(c(t))$, $M(t)=M(c(t)), F(t)=F(c(t))$ and $\tilde{F}(t)=F\left(c_{N(t), M(t)}\right)$. By Proposition 4.1, $F(t) \geqq \tilde{F}(t)$. With this notation we have:

\section{Proposition 4.2.}

$0 \leqq F(t)-\tilde{F}(t) \leqq[F(0)-\tilde{F}(0)] \exp \left[-\int_{0}^{t} d s M(s)\right]$.

This will be proved by the following inequality which appears to us to be new.

Proposition 4.3. Let $c(\cdot) \geqq 0$ and $c, c \ln c \in L^{1}([0, \infty))$ then

$$
\begin{aligned}
& \int_{0}^{\infty} \int_{0}^{\infty} d x d y c(x) c(y) \ln c(x+y) \\
& \quad \leqq \int_{0}^{\infty} d x c(x) \int_{0}^{\infty} d y c(y) \ln c(y)-\left[\int_{0}^{\infty} d x c(x)\right]^{2}
\end{aligned}
$$

(The equality is attained only by the exponential functions $e^{-\lambda x}$.)

Proof. Let $f(x)=\int_{x}^{\infty} d y c(y)$

$$
\begin{aligned}
& \int_{0}^{\infty} \int_{0}^{\infty} d x d y c(x) c(y) \ln c(x+y)-\int_{0}^{\infty} d x c(x) \int_{0}^{\infty} d y c(y) \ln c(y) \\
& \quad=\int_{0}^{\infty} d x c(x) f(x) \int_{0}^{\infty} d y c(x+y) / f(x)[c(y) / c(x+y)] \ln [c(x+y) / c(y)] .
\end{aligned}
$$


Applying Jensen's inequality for the semibounded concave function $a \mapsto a \ln a^{-1}$ we get for the above expression

$$
\begin{aligned}
& \leqq \int_{0}^{\infty} d x c(x) f(x)\left[\int_{0}^{\infty} d y c(y) / f(x)\right] \ln \left[f(x) / \int_{0}^{\infty} d y c(y)\right] \\
& =-\left[\int_{0}^{\infty} d x c(x)\right]^{2} \ln \int_{0}^{\infty} d y c(y)-\int_{0}^{\infty} d y c(y) \int_{0}^{\infty} d x\left[\frac{d}{d x} f(x)\right] \ln f(x) \\
& =-f^{2}(0) \ln f(0)-f(0)[f(x) \ln f(x)]_{0}^{\infty}+f(0) \int_{0}^{\infty} d x f(x) \frac{d}{d x} \ln f(x) \\
& =-\left[\int_{0}^{\infty} d x c(x)\right]^{2} \cdot
\end{aligned}
$$

Remark 4.1. The above inequality does not hold on $\mathbb{R}$, e.g. for $e^{-|x|}$, however a slightly weaker inequality does hold in $\mathbb{R}^{n}$. For $f \geqq 0$, and $f, f \ln f \in L^{1}(\mathbb{R})$

$$
\begin{aligned}
& \int_{-\infty}^{\infty} d^{n} x \int_{-\infty}^{\infty} d^{n} y f(y) \ln f(x+y) \\
& \leqq \int_{-\infty}^{\infty} d^{n} x f(x) \int_{0}^{\infty} d^{n} y f(y) \ln f(y)-\frac{1}{2} n \ln 2\left[\int_{-\infty}^{\infty} d x f(x)\right]^{2} .
\end{aligned}
$$

In fact, differentiating Young's inequality with its best constants (which were found by Beckner [6] and by Brascamp and Lieb [7]) one may get, for $f, g, h \geqq 0$ etc.,

$$
\begin{aligned}
& \int d^{n} x \int d^{n} y f(x) g(y) \ln h(x-y) \\
& \leq \\
& \quad \frac{1}{2} \int d^{n} x f(x) \ln f(x) \int d^{n} y g(y)+\frac{1}{2} \int d^{n} x f(x) \int d^{n} y g(y) \ln g(y) \\
& \quad+\int d^{n} x f(x) \int d^{n} y g(y)\left[\ln \int d^{n} z h(z)-\frac{1}{2} \ln \int d^{n} z f(z)-\frac{1}{2} \ln \int d^{n} z g(z)\right] \\
& \quad-n \frac{1}{2} \ln 2 \int d^{n} x f(x) \int d^{n} y g(y) .
\end{aligned}
$$

The equality is attained by (amon others) $f=g=e^{-2 x^{2}}, h=e^{-x^{2}}$.

We need the stronger inequality, (4.6), to control the decay of $F$.

Proof of Proposition 4.2. It was shown in the previous section that $c(t, \cdot)$ may be approximated by solutions of the modified Eq. (3.18) which are bounded and supported in $[0, z]$. For these functions $\frac{d F}{d t}$ is given by (3.32) which coincides with (3.14). It follows by the semi-continuity of $F$ that it is enough to prove the proposition for these functions, i.e. assuming that Eq. (3.14) is valid and that the four terms in the integrand in (3.14) are integrable. In such case [with $c(\cdot)=c(t, \cdot)$ ]

$$
\begin{aligned}
\frac{d}{d t} F(t)= & \int_{0}^{\infty} \int_{0}^{\infty} d x d y c(x) c(y) \ln c(x+y)-2 \int_{0}^{\infty} \int_{0}^{\infty} d x d y c(x) c(y) \ln c(y) \\
& +\int_{0}^{\infty} \int_{0}^{\infty} d x d y c(x) c(y)[c(x+y) / c(x) c(y)] \ln [c(x) c(y) / c(x+y)] .
\end{aligned}
$$


Applying the inequality (4.6) to the first term and Jensen's inequality for the concave function $a \mapsto a \ln a^{-1}$ to the last term in (4.9) we get $[$ since

$$
\begin{gathered}
\left.\int_{0}^{\infty} \int_{0}^{\infty} d x d y c(x+y)=N(c)\right] \\
\frac{d}{d t} F(t) \leqq-M(t) \int_{0}^{\infty} d y c(y) \ln c(y)-M(t)^{2}+N(t) \ln \left[M(t)^{2} / N(t)\right] \\
=-M(t) F(t)-2 M(t)^{2}+N(t) \ln \left[M(t)^{2} / N(t)\right] .
\end{gathered}
$$

But

$$
\tilde{F}(t)=M(t) \ln \left[M(t)^{2} / N(t)\right]-2 M(t)
$$

and, by (2.13)

$$
\frac{d}{d t} \tilde{F}(t)=\left[N(t)-M(t)^{2}\right] \ln \left[M(t)^{2} / N(t)\right] .
$$

(4.10)-(4.12) add exactly to:

$$
\frac{d}{d t}[F(t)-\tilde{F}(t)] \leqq-M(t)[F(t)-\tilde{F}(t)] .
$$

Thus

$$
\frac{d}{d t}[F(t)-\tilde{F}(t)] \exp \left[\int_{0}^{t} d s M(s)\right] \leqq 0
$$

which proves (4.5).

Remark 4.2. It is illuminating to follow the time evolution by observing the three thermodynamic quantities: $N(c), M(c)$, and $F(c)$. For a given value of $N(c)$, which is a constant of motion, the joint range of $(M(c), F(c))$ is the convex set defined by $M(c)>0, \hat{F}(c) \geqq \hat{F}(N(c), M(c))$. The extremal points of this set (and only those) correspond to unique functions $-c_{N, M}$ (which describe states of "quasi-equilibrium"). While $M$ undergoes an autonomous time evolution, $F$ decreases always faster than it does at the corresponding boundary point with the same $M$ (by Proposition 4.2). Thus a function with an extremal value of $(M, F)$ retains this property.

Indeed it may be verified directly that for any $N$ and $M(0)$,

$$
c(t, x)=c_{N, M(t)}(x)
$$

is a solution provided $M(t)$ obeys Eq. (2.13), i.e. $M(t)$ is of the form (2.16). Families of simple solutions which preserve a "canonical form" (in the terminology of [8]) and which exhibit convergence to equilibrium were found also for other related equations, e.g. one which describes a harmonic oscillator interacting with a heat bath [9].

Any solution $c(t)$ may, therefore, be compared to a solution with the same $N$ and $M(t)$ for which $F$ is minimized at all times. Furthermore, the difference in $F$ decays exponentially fast (see also Remark 6.3). 


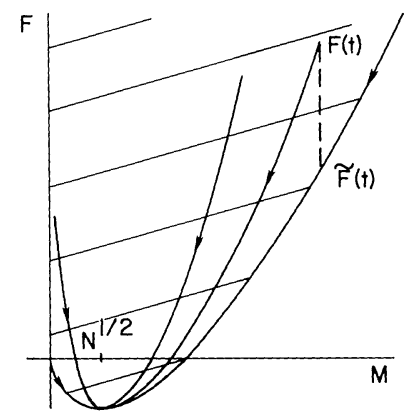

Fig. 1. The range and the time evolution of $(M(c), F(c))$

\section{Convergence to Equilibrium}

a) Boltzmann's Argument Made Precise

The decay of the free energy implies directly strong convergence to equilibrium.

Proposition 5.1. Let $c(t, \cdot)$ be a solution of $E q$. (2.1) with $c(0, \cdot) \in \mathscr{F}_{+}$and $F(c(0))<\infty$. Then

$$
\left\|c(t, \cdot)-c_{N, N^{1 / 2}}(\cdot)\right\|_{1} \underset{t \rightarrow \infty}{\longrightarrow} 0
$$

with $N=N(c)$.

To prove it we use the following estimate (see also remark [12]).

Lemma 5.1. $\forall \varepsilon>0 \exists a(\varepsilon)<\infty$ such that $\forall c \in \mathscr{F}_{+}$

$$
\left\|c-c_{N, M}\right\|_{1} \leqq \varepsilon M+a(\varepsilon)\left[F(c)-F\left(c_{N, M}\right)\right]
$$

with $M=M(c)$ and $N=N(c)$.

Proof. Let $g(u)=u \ln u+1-u$. Since $g$ is strictly convex and $g(1)=g^{\prime}(1)=0, \forall \varepsilon>0$ $\exists a(\varepsilon)<\infty$ such that

$$
\forall u \geqq 0:|u-1| \leqq \varepsilon+a(\varepsilon) g(u) .
$$

(5.2) follows by substituting $u=c(x) / c_{N, M}(x)$ and integrating (5.3) with $\int_{0}^{\infty} d x c_{N, M}(x)$

Proof of Proposition 5.1. Let $N=N(c)$ and $M(t)=M(c(t))$. By Lemma 5.1 and Proposition 4.2, for any $\varepsilon>0$

$$
\begin{aligned}
& \left\|c(t, \cdot)-c_{N, N^{1 / 2}}(\cdot)\right\|_{1} \leqq\left\|c(t, \cdot)-c_{N, M(t)}(\cdot)\right\|_{1} \\
& \quad+\left\|c_{N, M(t)}(\cdot)-c_{N, N^{1 / 2}}(\cdot)\right\|_{1} \\
& \quad \leqq \\
& \quad \varepsilon M(t)+a(\varepsilon) \exp \left[-\int_{0}^{x} d s M(s)\right]\left[F(c(0))-F\left(c_{N, M(0)}\right)\right] \\
& \quad+\left\|c_{N, M(t)}(\cdot)-c_{N, N^{1 / 2}}(\cdot)\right\|_{1}
\end{aligned}
$$

which proves (5.1). 


\section{b) A Stronger Result}

The only restrictive assumption in Proposition 5.1 is that $F(c(0))<\infty$. This may be removed by an additional argument, leading to:

Proposition 5.2. There exists a function $Q(t, N)$, with

$$
\lim _{t \rightarrow \infty} Q(t, N)=0 \quad \forall N>0,
$$

such that for any solution of Eq. (2.9) in $\mathscr{F}_{+}$:

$$
\left\|c(t, \cdot)-c_{N, N^{1 / 2}}(\cdot)\right\|_{1} \leqq Q(t, N)
$$

for $N=N(c(0))$.

The proof is based on the following three lemmas.

Lemma 5.2. There exists a function $T_{1}(\varepsilon, N, F)<\infty$ such that for any solution in $\mathscr{F}_{+}$of Eq. (2.9) with $N(c)=N$ and $F(c(0)) \leqq F$ :

$$
\left\|c(t, \cdot)-c_{N(c), N(c)^{1 / 2}}(\cdot)\right\|_{1} \leqq \varepsilon \quad \forall t \geqq T_{1}(\varepsilon, N, F) .
$$

Proof. This lemma is directly implied by the inequality (5.4) and by:

$$
\left|M(c(t))-N^{1 / 2}(c)\right| \leqq N^{1 / 2}\left[\operatorname{coth}\left(N^{1 / 2} t\right)-\tanh \left(N^{1 / 2} t\right)\right]
$$

which follows from (2.16).

Lemma 5.3. For any $\varepsilon, T, N, M>0 \exists \delta=\delta(\varepsilon, T, M, N)$ such that if $c_{1}(t, x), c_{2}(t, x)$ are two solutions, in $\mathscr{F}_{+}$, of Eq. (2.9) which satisfy:

$$
\begin{aligned}
& \left\|c_{i}(0)\right\|_{x} \leqq N, \quad\left\|c_{i}(0)\right\|_{1} \leqq M \quad i=1,2 \\
& \left\|c_{1}(0)-c_{2}(0)\right\|<\delta
\end{aligned}
$$

then

$$
\left\|c_{1}(t)-c_{2}(t)\right\|<\varepsilon \quad \forall t \in[0, T] .
$$

Proof. $c_{1}$ can be obtained from $c_{2}$ by repeatedly applying the Picard mapping (2.12). To prove the lemma it suffices to show that for any $N, M>0$ there is $T>0$ for which the claim holds, and it is convenient to choose $T$ so that the Picard mapping is a contraction on the space of functions

$$
c:[0, T] \rightarrow\left\{f \in \mathscr{F}_{+} \mid N(f) \leqq N, M(f) \leqq M\right\},
$$

as in the proof of Lemma 2.2. For such $T$ the claim follows from the invariance of $c_{2}$ under the Picard mapping which corresponds to $c_{2}(0, \cdot)$, by an argument which was used in the proof of Lemma 3.3.

Lemma 5.4. $\forall N>0 \exists G(N)<\infty$ and $B(t, N)$, with

$$
\lim _{t \rightarrow \infty} B(t, N)=0 \text {, }
$$

such that any solution of Eq. (2.9) admits a decomposition

$$
c(t, x)=b(t, x)+g(t, x)
$$


for which

i) $b(t, x), g(t, x) \geqq 0 \quad \forall t, x \geqq 0$,

ii) $\|b b(t, \cdot)\| \mid \leqq B(t, N) \quad \forall t>0$,

iii) $\|g(t, \cdot)\|_{\infty} \equiv \sup \{g(t, x) \mid x \in[0, \infty)\}<G(N(c)) \quad \forall t \geqq 0$.

Proof. Let $c(t, x)$ be a solution of (2.9) in $\mathscr{F}_{+}$and let

$$
N=N(c(0)), \quad M(t)=M(c(t)) .
$$

By (5.8), $\forall N \exists t_{0}(N)<\infty$ such that $\forall t \geqq t_{0}(N)$

$$
(3 / 4) N^{1 / 2} \leqq M(t) \leqq(5 / 4) N^{1 / 2} .
$$

It is therefore enough to find $B$ and $G$ for which the claim is satisfied on the restricted class of functions $c$ for which (5.10) holds $\forall t \geqq 0$.

According to the explicit solution (2.8)

$$
\alpha_{t} f=\alpha_{t}^{(1)} f+\alpha_{t}^{(2)} f
$$

with

$$
\begin{aligned}
& \left(\alpha_{t}^{(1)} f\right)(x)=e^{-t x} f(x) \\
& \left(\alpha_{t}^{(2)} f\right)(x)=2 t e^{-t x} \int_{0}^{\infty} d y f(y)+t^{2} e^{-t x} \int_{0}^{\infty} d y(y-x) f(y) .
\end{aligned}
$$

The only use which will be made of the explicit formula for $\alpha_{t}^{(2)}$ is to derive:

$$
\left\|\alpha_{t}^{(1)} f\right\|_{\infty} \leqq\|f\|_{\infty},\left\|\alpha_{t}^{(2)} f\right\|_{\infty} \leqq 2 t\|f\|_{1}+t^{2}\|f\|_{x} .
$$

The main idea of the proof is to decompose $c$ into solutions of the following equations

$$
\begin{aligned}
b(t, \cdot)= & \exp \left[-2 \int_{0}^{t} d u M(u)\right] \alpha_{t}^{(1)} c(0, \cdot) \\
& +\int_{0}^{t} d s \exp \left[-2 \int_{0}^{t} d u M(u)\right] \alpha_{t-s}^{(1)}[b(s, \cdot) * b(s, \cdot)]
\end{aligned}
$$

and

$$
\begin{aligned}
g(t, \cdot)= & \exp \left[-2 \int_{0}^{t} d u M(u)\right] \alpha_{t}^{(2)} c(0, \cdot) \\
& +\int_{0}^{t} d s \exp \left[-2 \int_{0}^{t} d u M(u)\right] \alpha_{t-s}^{(2)}[c(s, \cdot) * c(s, \cdot)] \\
& +\int_{0}^{t} d s \exp \left[-2 \int_{0}^{t} d u M(u)\right] \alpha_{t-s}^{(1)}\{g(s, \cdot) *[c(s, \cdot)+b(s, \cdot)]\},
\end{aligned}
$$

which add up to the Eq. (2.15) for $c(t, x)$.

The local existence and uniqueness of a solution of (5.13) in $\mathscr{F}_{+}$can be easily seen by a contraction mapping argument similar to the one used to prove Lemma 
2.2 and Proposition 2.3. In order to prove the decay of $b(t, \cdot)$ it is convenient to look at

$$
\hat{b}(t, x)=e^{t x} b(t, x)
$$

which satisfies

$$
\begin{aligned}
\hat{b}(t, \cdot)= & \exp \left[-2 \int_{0}^{t} d u M(u)\right] c(0, \cdot) \\
& +\int_{0}^{t} d s \exp \left[-2 \int_{0}^{t} d u M(u)\right] \hat{b}(s, \cdot) * \hat{b}(s, \cdot) .
\end{aligned}
$$

By the above mentioned argument, Eq. (5.16) has a unique $\|\cdot\|_{1}$-continuous solution. While it is not obvious from $(5.15)$ that $\|\hat{b}(t, \cdot)\|_{1}<\infty, \hat{b}$ has to be that solution by the uniqueness of the solution of (5.13). It follows that $\|\hat{b}(t, \cdot)\|_{1}$ is differentiable and satisfies:

$$
\left\{\begin{array}{l}
\frac{d}{d t}\|\hat{b}(t, \cdot)\|_{1}=-2 M(t)\|\hat{b}(t, \cdot)\|_{1}+\|\hat{b}(t, \cdot)\|_{1}^{2} \\
\|\hat{b}(0, \cdot)\|_{1}=M(0) .
\end{array}\right.
$$

This implies

$$
\|\hat{b}(t, \cdot)\|_{1} \leqq M(t)
$$

since $\frac{d}{d t}\|\hat{b}(t, \cdot)\|_{1}<\frac{d}{d t} M(t)$ whenever $\|\hat{b}(t, \cdot)\|_{1}=M(t)$.

By (5.10) and (5.17)

$$
\|\hat{b}(t, \cdot)\|_{1} \leqq(5 / 4) N^{1 / 2}
$$

and

$$
\begin{aligned}
\frac{d}{d t}\|\hat{b}(t, \cdot)\|_{1} \leqq & -2(3 / 4) N^{1 / 2}\|\hat{b}(t, \cdot)\|_{1} \\
& +(5 / 4) N^{1 / 2}\|\hat{b}(t, \cdot)\|_{1}=-4^{-1} N^{1 / 2}\|\hat{b}(t, \cdot)\|_{1},
\end{aligned}
$$

for $t=0$ and hence for all $t \geqq 0$. Therefore

$$
\|\hat{b}(t, \cdot)\|_{1} \leqq M(0) \exp \left[-4^{-1} N^{1 / 2} t\right],
$$

yielding

$$
\begin{aligned}
\|b(t, \cdot)\| & <\|\hat{b}(t, \cdot)\|_{1} \sup \left\{(1+x) e^{-t x}\right\} \\
& <(5 / 4) N^{1 / 2}\left(1+t^{-1}\right) \exp \left[-4^{-1} N^{1 / 2} t\right]
\end{aligned}
$$

which proves ii) [for the class restricted by $(5.10)]$ with $B(t, N)$ defined by the last expression.

For any solution of Eq. (5.14)

$$
\sup _{t \geqq 0}\|g(t, \cdot)\| \leqq A+D \sup _{t \geqq 0}\|g(t, \cdot)\|_{\infty}(\leqq \infty)
$$


with

$$
\begin{aligned}
A= & \sup _{t \geqq 0}\left\{\left[2 t N^{1 / 2}+t^{2}(5 / 4) N^{1 / 2}\right] \exp \left[-2(3 / 4) N^{1 / 2} t\right]\right\} \\
& +\int_{0}^{\infty} d u\left[2 u N^{1 / 2}+2 u^{2}(5 / 4) N^{1 / 2}\right]^{2} \exp \left[-2(3 / 4) N^{1 / 2} u\right]<\infty
\end{aligned}
$$

and

$$
\begin{aligned}
D & =\sup _{t>0}\left\{\int_{0}^{t} d s\left[M(s)+\|b(s, \cdot)\|_{1}\right] \exp \left[-2 \int_{s}^{t} d u M(u)\right]\right\} \\
& =\sup _{t>0}\left\{\int_{0}^{t} d s\left[M(t-s)+\|b(t-s, \cdot)\|_{1}\right] \exp \left[-2 \int_{0}^{t} d u M(t-u)\right]\right\}
\end{aligned}
$$

(by 5.12). The last identity was written to facilitate the limit $t \rightarrow \infty$. Since $\|b(s, \cdot)\|_{1} \leqq M(s)$ and

$$
\int_{0}^{t} d s M(t-s) \exp \left[-2 \int_{0}^{s} d u M(t-u)\right]=\frac{1}{2}
$$

$D$ is the supremum of a continuous function which is bounded by 1 and equals 0 at 0 and $\frac{1}{2}$ at $\infty$. Thus

$$
D<1 \text {. }
$$

Using (5.27) in the standard constructive argument which was refered to before, one can easily prove the existence, uniqueness and positivity of a solution of Eq. (5.14). It satisfies

$$
\sup _{t>0}\left\{\|g(t, \cdot)\|_{\infty}+\|g(t, \cdot)\| \|\right\}<\infty,
$$

since one gets a similar inequality to (5.23) for the $|\|\cdot\|| \mid$-norm, with the same $D$ and a modified but finite $A$.

Therefore $b(t, x)+g(t, x)$ is a solution of Eq. (2.15) in $\mathscr{F}_{+}$which, by the uniqueness, implies (5.9).

Finally, (5.23), (5.27), and (5.28) imply

$$
\sup _{t>0}\|g(t, \cdot)\|_{\infty} \leqq A /(1-D) \text {, }
$$

proving iii) with $G(N)=A /(1-D)$.

Proof of Proposition 5.2. It is enough to show that for any $\varepsilon, N>0 \exists T=T(\varepsilon, N)$ such that for any solution in $\mathscr{F}_{+}$of Eq. (2.9) with $N(c)=N$

$$
\left\|c(t, \cdot)-c_{N, N^{1 / 2}}(\cdot)\right\|_{1} \leqq \varepsilon \quad \forall t \geqq T(\varepsilon, N) .
$$

Let $\varepsilon, N$ be given and let $T_{1}=T_{1}\left(\varepsilon, N,(5 / 4) N^{1 / 2} \ln G(N)\right)$, with the notation of Lemmas 5.2-5.4. By Lemma 5.4 $\exists T_{2} \in\left[t_{0}(N), \infty\right)\left[t_{0}\right.$ is defined by (5.10)], such that $\forall t \geqq T_{2}$ :

$$
2 B(t, N) \leqq \delta\left(\varepsilon, T_{1}, N, 10 / 4 N^{1 / 2}\right) .
$$


We define $T$ by:

$$
T(\varepsilon, N)=T_{1}+T_{2} \text {. }
$$

For any $c(t, x)$ as above and $t \geqq T(\varepsilon, N)$ one may find, by Lemma 5.4 , a function $\bar{g}_{t-T_{1}}(x) \geqq 0$ such that

$$
\begin{aligned}
& \left\|c\left(t-T_{1}, \cdot\right)-\bar{g}_{t-T_{1}}(\cdot)\right\| \| \leqq 2 B\left(t-T_{1, N}\right) \\
& \left\|\bar{g}_{1-T_{1}}(\cdot)\right\|_{\infty} \leqq G(N),
\end{aligned}
$$

and

$$
N\left(g_{t-T_{1}}\right)=N(c), M\left(\bar{g}_{t-T_{1}}\right)<(10 / 4) N^{1 / 2}
$$

[for the last inequality use is made of: $t-T_{1} \geqq T_{2} \geqq t_{0}(N)$ ]. We approximate $c(t, \cdot)$ by $\bar{g}_{t-T_{1}}\left(T_{1}, \cdot\right)$ which is obtained evolving from $\bar{g}_{t-T_{1}}(\cdot)$ for the time $T_{1}$, by Eq. (2.9). By Lemma 5.2

$$
\left\|\bar{g}_{t-T_{1}}\left(T_{1}, \cdot\right)-c_{N, N^{1 / 2}}(\cdot)\right\|_{1}<\varepsilon
$$

and by Lemma 5.3

$$
\left\|c(t, \cdot)-\bar{g}_{t-T_{1}}\left(T_{1}, \cdot\right)\right\|_{1}<\varepsilon,
$$

since

$$
\|\| c\left(t-T_{1}, \cdot\right)-\bar{g}_{t-T_{1}}(\cdot) \|<\delta\left(\varepsilon, T_{1} N,(10 / 4) N^{1 / 2}\right) .
$$

(5.30) follows now by adding (5.34) and (5.35).

\section{The Linearized Equation}

In order to study the time evolution flow in the vicinity of any of the equilibria $c_{N, N^{1 / 2}}(x)=\exp \left(-N^{-1 / 2} x\right)$ it is convenient to change variable to $\varphi(\cdot)$ defined by:

$$
c(x)=\exp \left(-N^{-1 / 2} x\right)+\varphi(x) \exp \left(-N^{-1 / 2} x / 2\right) .
$$

The equation for $\varphi$ which corresponds to Eq. (1.5) is

$$
\begin{aligned}
\frac{\partial}{\partial t} \varphi(t, x)= & 2 \int_{0}^{\infty} d y e^{-N-1 / 2|x-y| / 2} \varphi(y) \\
& -2 \int_{0}^{\infty} d y e^{-N^{-1 / 2}(x+y) / 2} \varphi(y)-\left(2 N^{1 / 2}+x\right) \varphi(x) \\
& +(\varphi * \varphi)(x)-2 \varphi(x) \int_{0}^{\infty} d y e^{-N^{-1 / 2} y / 2} \varphi(y) .
\end{aligned}
$$

In the vicinity of the equilibrium $c_{N, N^{1 / 2}}$, described by

$$
\|\varphi\|_{2} \equiv\left(\int_{0}^{\infty} d x|\varphi(x)|^{2}\right)^{1 / 2} \ll N^{1 / 2},
$$


the non-linear terms are of second order, leaving us with the linearized generator $L_{N}$ defined by:

$$
\begin{aligned}
\left(L_{N} \varphi\right)(x)= & 2 \int_{0}^{\infty} d y e^{-N^{-1 / 2}|x-y| / 2} \varphi(y) \\
& -2 \int_{0}^{\infty} d y e^{-N^{-1 / 2}(x+y) / 2} \varphi(y)-\left(2 N^{1 / 2}+x\right) \varphi(x) .
\end{aligned}
$$

The local behaviour of a flow near a stationary point depends on the spectrum of the linearized generator. The spectra of $L_{N}$ can be computed exactly and are described in the following result.

Proposition 6.1. For $N>0$ :

i) $L_{N}$ are self adjoint operators on $L^{2}([0, \infty))$.

ii) $N^{-1 / 2} L_{N}$ are unitarily equivalent.

iii) The spectrum of $L_{N}$ is $\left(-\infty,-2 N^{1 / 2}\right] \cup\{0\}$. On $\left(-\infty,-2 N^{1 / 2}\right)$ the spectral measure is absolutely continuous with respect to the Lebesgue measure, with multiplicity 1 , and at $-2 N^{1 / 2}$ and 0 it has two, non-degenerate, eigenvalues.

iv) If $\varphi \in L^{2}([0, \infty))$ corresponds to $c$ with $N(c)=N$, then $\varphi$ is orthogonal to the 0-eigenvector of $L_{N}$.

Proof. The sum of the first two terms in the r.h.s. of (6.4) can be written as an integral over $\mathbb{R}$ of the antisymmetric function $\operatorname{sgn}(x) \varphi(|x|)$ with the translation invariant kernel $2 e^{-N^{-1 / 2}|x-y| / 2}$. It follows that

$$
L_{N}=\frac{8 N^{1 / 2}}{1+4 N p^{2}}-\left(2 N^{1 / 2}+x\right),
$$

where $-p^{2}$ is the Laplacian with the Dirichlet boundary condition and $\left(2 N^{1 / 2}+x\right)$ is a multiplication operator.

Since the first term in (6.5) is a bounded operator and the second a selfadjoint one, $L_{N}$ is selfadjoint on the domain of $x . N^{-1 / 2} L_{N}$ is unitarily equivalent to $L_{1}$ by the dilation: $\varphi(x) \rightarrow N^{1 / 4} \varphi\left(x N^{1 / 2}\right)$.

Thus i) and ii) are proven. By ii), to prove iii) and iv) it is enough to describe the spectrum of $L \equiv L_{1}$. This is done by the following two lemmas.

Lemma 6.1. L has exactly two eigenvectors in $L^{2}([0, \infty))$. The corresponding eigenvalues are 0 and -2 .

Proof.

$$
\varphi_{0}(x)=x e^{-x / 2}
$$

is a 0 -eigenvector, as may be guessed from the time invariance of

$$
\int_{0}^{\infty} d x x c(x)=1+\int_{0}^{\infty} d x x e^{-x / 2} \varphi(x)
$$

(Notice that iv) is satisfied.) 
Let now $\varphi_{\lambda}$ be a $\lambda$-eigenvector. Then $\varphi_{\lambda}$ is in the domain of $x$, which coincides with that of $L$, and

$$
8 /\left(1+4 p^{2}\right) \varphi_{\lambda}-(2+x) \varphi_{\lambda}=\lambda \varphi_{\lambda}
$$

Therefore $\exists f_{\lambda} \in L^{2}([0, \infty))$ such that

$$
f_{\lambda}=8 /\left(1+4 p^{2}\right) \varphi_{\lambda}=(x+\lambda+2) \varphi_{\lambda},
$$

which implies

$$
\left(1+4 p^{2}\right) f_{\lambda}=8 \varphi_{\lambda}=8 /(x+\lambda+2) f_{\lambda} .
$$

Thus the function

$$
g(x)=f_{\lambda}(x-(\lambda+2))
$$

has an absolutely continuous derivative and solves the eigenvalue problem, in $L^{2}([\lambda+2, \infty))$,

$$
(-4 \Delta-8 / x) g(x)=-g(x)
$$

with the boundary condition:

$$
g(\lambda+2)=0 \text {. }
$$

By a standard argument, which uses the constancy of the Wronskian of the two solutions [10], the Schrödinger equation (6.12) has at most one solution which is not divergent at $\infty$. This solution may be found from the known eigenvector $\varphi_{0}$. It is :

$$
g(x)=(x-2) x e^{-(x-2) / 2} .
$$

The lemma follows now, using (6.13), from the number and location of the zeroes of $g$. The (-2)-eigenvector of $L$ is

$$
\varphi_{-2}(x)=(x-2) e^{-x / 2} \text {. }
$$

Lemma 6.2. On the orthogonal complement of $\left\{\varphi_{0}, \varphi_{-2}\right\}$ the spectrum of $L$ is $(-\infty, 2]$ and the spectral measure is absolutely continuous with multiplicity 1.

Proof. We may use the former approach to find also generalized eigenvalues. For distributions, however, (6.9) does not imply the last equality in (6.10). Consequent$1 y,(6.12)$ is replaced by

$$
(-4 \Delta-8 / x) g(x)=-g(x)+\alpha \delta(x),
$$

with an undetermined $\alpha$. In addition to (6.14), (6.16) admits the solution

$$
\hat{g}(x)= \begin{cases}(x-2) x e^{-(x-2) / 2} & x \geqq 0 \\ 0 & x \leqq 0\end{cases}
$$

To the zeroes of $\bar{g}$ correspond the following generalized eigenvectors

$$
\begin{aligned}
\varphi_{\lambda}(x) & =8^{-1}(1-4 \Delta) \hat{g}(x+\lambda+2) \\
& =\delta(x+\lambda+2)-(x+\lambda) e^{-(x+\lambda) / 2} \theta(x+\lambda+2)
\end{aligned}
$$

with $\lambda \in(-\infty,-2)$. Here $\theta(x) \equiv(1+\operatorname{sgn} x) / 2$. 
Although $\varphi_{i}$ were found by an uncomplete argument, the following orthonormality and completeness relations can be verified directly, proving Lemma 6.2.

$$
\int_{0}^{\infty} d x \bar{\varphi}_{\lambda_{1}}(x) \varphi_{\lambda_{2}}(x)=\delta\left(\lambda_{1}-\lambda_{2}\right)
$$

for $\lambda_{1} \in(-\infty,-2), \lambda_{2} \in(-\infty,-2) \cup\{-2,0\}$, and

$$
\int_{-\infty}^{-2} d \lambda \bar{\varphi}_{\lambda}(x) \varphi_{\lambda}(y)+\varphi_{-2}(x) \varphi_{-2}(y)+\varphi_{0}(x) \varphi_{0}(y)=\delta(x-y) .
$$

Remark 6.1. That the essential spectrum of $L$ is $(-\infty,-2]$ may be seen without diagonalizing $L$ using, instead, Weyl's theorem [10], since $\left(1+4 p^{2}\right)^{-1}$ is a relatively compact perturbation of $-(2+x)$.

Remark 6.2. The kernel of the linearized semigroup can be obtained explicitly using (6.19) and (6.20). For the discrete case this was done, independently, by Kjær [4] who used it to study the approach to equilibrium.

Remark 6.3. In the formal expansion of $F(c)$ in $\varphi$, defined by 6.1 with $N=N(c)$. the linear term vanishes and

$$
F(c) \approx F\left(c_{N, N^{1 / 2}}\right)+\frac{1}{2} \int_{0}^{\infty} d x|\varphi(x)|^{2} .
$$

The spectral analysis suggests that whenever (6.21) is a valid approximation and $M(c(0, \cdot)) \neq N(c)^{1 / 2}$ then

$$
\left|F(c(t, \cdot))-F\left(C_{N, N^{1 / 2}}(\cdot)\right)\right| /\left[M(c(t, \cdot))-N^{1 / 2}\right]^{2} \underset{t \rightarrow \infty}{\longrightarrow} N^{-1 / 2} .
$$

\section{Appendix: An Exact Solution for the Laplace Transform}

The Laplace transform, $\gamma(t, y)=\int_{0}^{\infty} e^{-x y} c(t, x) d x$, may be expressed in a closed form. Equation (1.5) transforms to

$$
\frac{\partial \gamma}{\partial t}=2 \frac{\gamma(t, 0)-\gamma(t, y)}{y}+\frac{\partial \gamma}{\partial y}+\gamma^{2}-2 \gamma(t, y) \gamma(t, 0) .
$$

$\gamma(t, 0)$ equals $M(c(t))$ given by Eq. (2.16). Setting $\chi(t, y)=\gamma(t, y)-\gamma(t, 0)$ we obtain

$$
\frac{\partial \chi}{\partial t}-\frac{\partial \chi}{\partial y}=\frac{2}{y} \chi-\chi^{2}-1
$$

The left hand side is the derivative of $\chi$ along $y+t=$ const, and along this line the equation therefore is a Riccati equation. Using a standard substitution for Riccati equations we set $\chi=\frac{y}{y+1}+\frac{1}{g(t, y)}$, obtaining

$$
\partial t-\frac{\partial g}{\partial y}=\left(\frac{2}{y}-\frac{2 y}{y+1}\right) g-1 \text {. }
$$


The solution is

$$
g(t, y)=\frac{y^{2}-1}{2 y^{2}}-e^{-2 y}\left(\frac{y+1}{y}\right)^{2} f(y+t)
$$

where $f$ is an arbitrary function of one variable. To determine $f$ we consider $\lim _{t \rightarrow 0} \gamma(t, y)$. We have

$$
\gamma(t, y)=\gamma(t, 0)-\frac{y}{y+1}+\frac{2 y^{2}}{y^{2}-1+2 e^{-2 y}(y+1)^{2} f(y+t)} .
$$

Hence for $t \rightarrow 0$ we get

$$
f(y)=\lim _{t \rightarrow 0} f(y+t)=\frac{\left(\frac{y}{y+1}\right)^{2} e^{2 y}}{\gamma(0, y)-\gamma(0,0)+\frac{y}{y+1}}+\frac{1}{2} \frac{1-y}{1+y} e^{2 y}
$$

and substituting in Eq. (A5) we obtain an expression for $\gamma(t, y)$ in terms of $\gamma(0, t+y)$ and $\gamma(0,0)$. This expression, however, does not readily lend itself to transformation back to $c(t, x)$.

Acknowledgments. It is our pleasure to thank E. H. Lieb, O. E. Lanford and N. G. van Kampen, who suggested the solution for the Laplace transform, for very stimulating discussions. M. A. is very grateful for the hospitality at the Chemistry Laboratory III of H. C. Ørsted Institute and TAB for the hospitality at the Department of Physics, Princeton University.

\section{References}

1. Blatz, P.J., Tobolsky, A.V.: J. Phys. Chem. 49, 77 (1945)

2. Flory, P.J.: J.A.C.S. 58, 1877 (1936)

3. Bak, T.A., Bak, K.: Acta Chem. Scand. 13, 1997 (1959)

4. Kjær, K.: To be published

5. Rassing, J.: Adv. Mol. Relax. Proc. 4, 55 (1972)

6. Beckner, W.: Ann. Math. 102, 159 (1975)

7. Brascamp, H.J., Lieb, E.H.: Adv. Math. 20, 151 (1975)

8. Andersen, H.C., Oppenheim, I., Shuler, K.E., Weiss, G.H.: J. Math. Phys. 5, 522 (1964)

9. Bak, T.A., Sørensen, P.G.: Adv. Chem. Phys. XV, 219 (1969)

10. Reed, M., Simon, B. : Methods of modern mathematical physics. New York: Academic Press (Vol. II) 1975 ; (Vol. IV) 1978

11. A mathematical treatment of the homogeneous Boltzmann equation can be found in Arkeryd, L: On the Boltzmann equation, I, II. Arch. Rat. Mech. Anal. 45, I-1, II-17 (1972)

12. Exponential decay in (5.1) can be proved using instead $\left\|C-C_{N . M}\right\|_{1}^{2} \leqq 4 e M\left[F(C)-F\left(C_{N . M}\right)\right]$, which follows from (9a) in H. P. McKean: Arch. Rat. Mech. Anal. 21, 343 (1966)

Communicated by J.L. Lebowitz 